REVISTA PROYECCIONES: 57-76.

JORNADAS MATEMATICAS, AGOSTO 1983.

\title{
BIFURCACIONES EN PROBLEMAS NO LINEALES
}

\author{
Dr. JAIME FIGUEROA NIETO*
}

Con el objeto de ilustrar de manera simple los problemas relativos a la teoría de bifurcación, consideremos los ejemplos siguientes:

EJEMPLO 1 (de Euler)

Uno de los primeros estudios de bifurcación se debe a Leonard Euler (1744) quién trató la deformación de una barra sometida a compresión axial (lo que se conoció como Elástica).

Él consideró el PVF :

$$
\mid \begin{aligned}
& \theta^{\prime \prime}+\lambda \sin \theta=0 \\
& \theta^{\prime}(0)=\theta^{\prime}(1)=0
\end{aligned}
$$

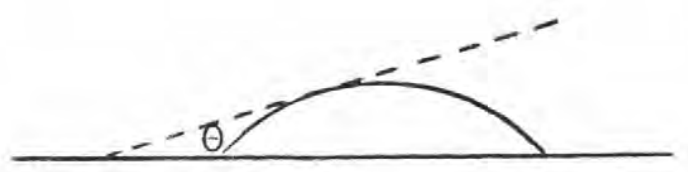

en que $\theta$ es el ángulo entre la tangente a la barra y el eje real

$\lambda$ es el esfuerzo aplicado

1 la longitud de la barra

* Profesor Departamento de Matemática y Ciencia de la Computación. Facultad de Ciencia. Universidad de Santiago de Chile. 
La solución trivial $\theta \equiv$ es solución del problema, $\forall \lambda \varepsilon \mathbf{R}$ Esto significa que la barra permanece derecha en equilibrio.

Sin embargo, suponiendo que $\theta$ es suficientemente pequeño, el problema se puede mirar como

$$
\mid \begin{aligned}
& \theta^{\prime \prime}+\lambda \theta=0 \\
& \theta^{\prime}(0)=\theta^{\prime}(1)=0
\end{aligned}
$$

que tiene por solución a $\theta(\lambda)=\varepsilon \cos \sqrt{\lambda} t$ con $|\varepsilon|$ suficientemente chico y $\lambda=\pi^{2}$. Esto es, si $\lambda$ excede $\pi^{2}$ una nueva familia de soluciones aparece y la barra se deforma.

Se puede dibujar el estado del sistema, por ejemplo dando $\theta(0)$ versus $\lambda$.

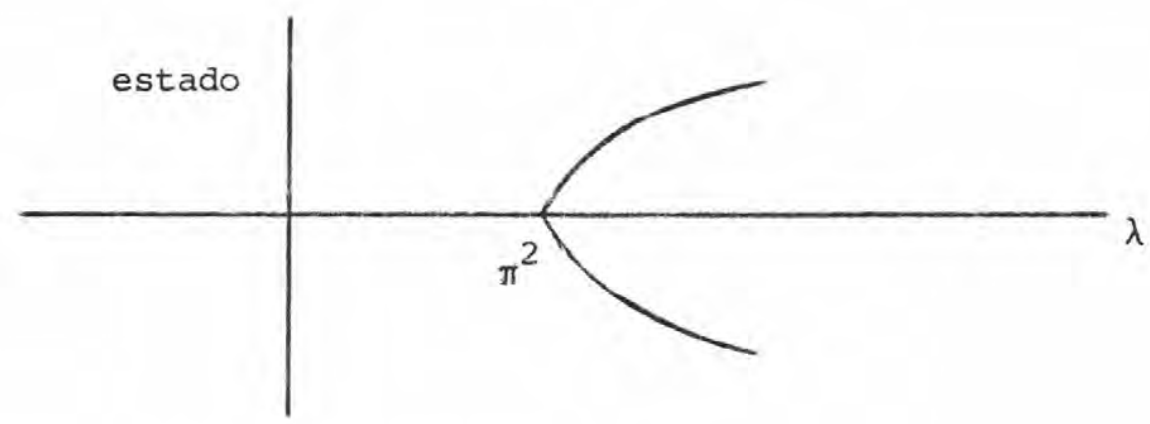

EJEMPLO 2 (Debido a E. Calabi)

Consideremos el siguiente sistema constituido por un aro con una ranura por donde puede moverse una bolita, aro sujeto a una barra por un hilo.

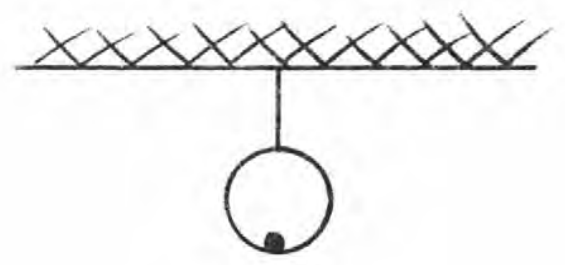


Se hace girar el aro con una velocidad angular $\omega$. Si $\omega$ es pe queña y la bolita ha sido desplazada, ella volverá a la posición de equilibrio. Si $\omega$ es suficientemente grande la bolita se ubicará en un punto distinto dependiendo de $\omega$.

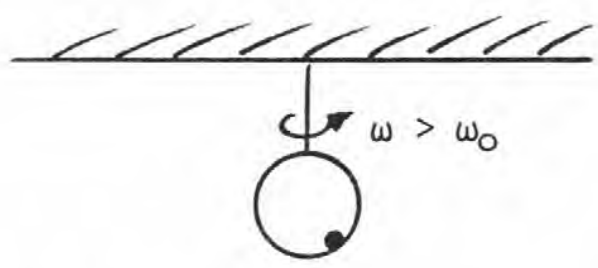

Si a partir de este nuevo punto se la mueve un poco, nuevamente volverá al punto.

Las posiciones de la bolita (soluciones del sistema) se encuen tran en curvas sobre un cilindro:

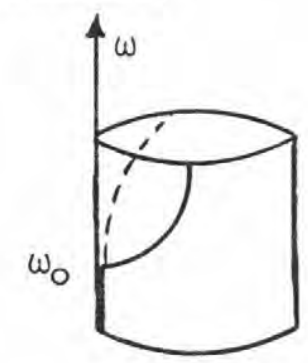

ENEMPLO 3 (Derivado del de E. Calabi)

otro tipo de bifurcación es la de un sistema consistente en una bolita dentro de una esfera. Las bifurcaciones se ilustran en la siguiente figura
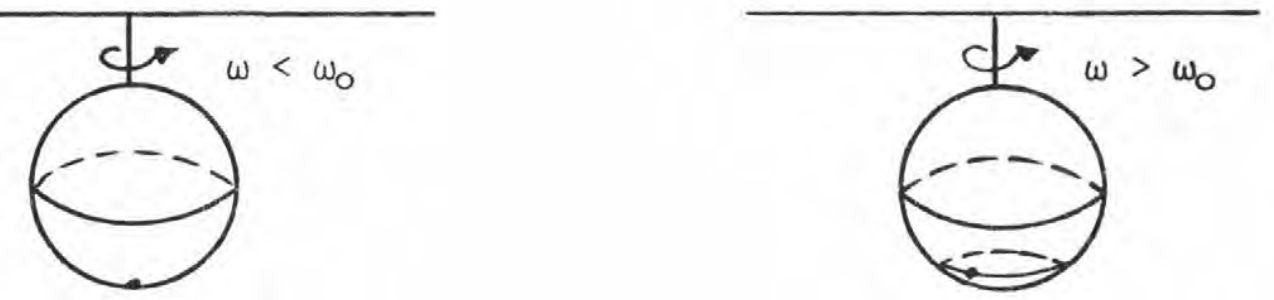

Es la bifurcación a órbitas periódicas. 


\section{FORAULACION ABSTRACTA.}

Sea $\mathrm{X}, \mathrm{Y}$ dos espacios de Banach y $\mathrm{G}: \mathrm{X} \longrightarrow \mathrm{Y}$ una aplicación de $X$ en $Y$. Sea $Z$ una curva de ceros de $G$. Un punto interior $\omega \in Z$ se llama un punto de bifurcación para $\mathrm{G}$ con respecto a $\mathrm{z}$ si cada vecindad de $\omega$ en $X$ contiene soluciones de $G(x)=0$ que no están en $Z$.

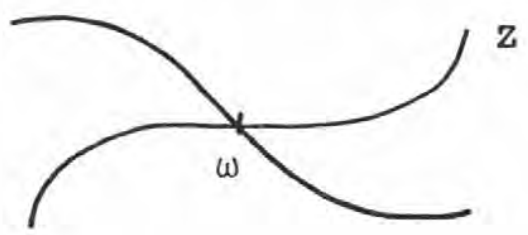

\section{OBSERVACION .}

Usualmente $\mathrm{x} C \operatorname{IR} \mathrm{x} \tilde{\mathrm{x}}$ y $\mathrm{z}=\mathbb{I} \times\{0\}$

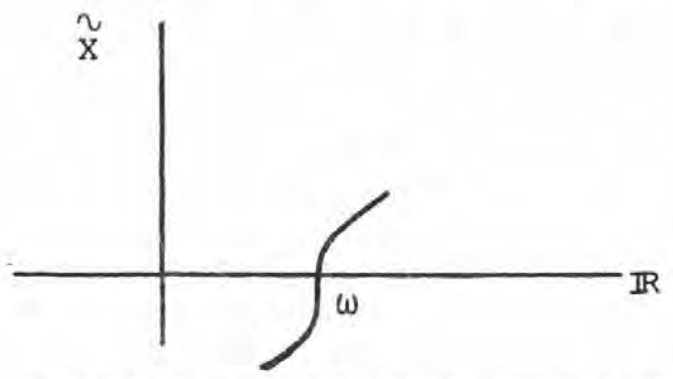

En este caso las soluciones son de la forma $(\lambda, \underset{\sim}{x})$ en que $\lambda$ es un parámetro de bifurcación usualmente con un significado físico tal como temperatura, magnitud de una fuerza, viscosidad, etc. Los elementos de $\mathrm{z}$ se llaman ceros triviales de G. Interesa conocer las soluciones no triviales que bifurcan de $\mathrm{z}$.

\section{ORIENTACION .}

Se presentará a continuación una teoría de bifurcación que estudia la multiplicidad de las soluciones reales de la ecuación de operadores no lineales $\mathrm{A} U=\cdot \lambda \mathrm{B}$ y la dependencia de estas soluciones del 
parámetro real $\lambda$.

DEFINICION.

Se dice que la ecuación $\mathrm{A} u=\lambda \mathrm{B} u$ tiene un punto de bifurcación en $\lambda=\lambda_{0}$ si la ecuación tiene al menos dos soluciones distintas $u_{1}(\lambda)$ y $u_{0}(\lambda)$ para $\lambda$ en una vecindad de $\lambda_{0}$ de tal manera que tanto $u_{1}(\lambda)$ como $u_{0}(\lambda)$ tiendan a $u_{0}\left(\lambda_{0}\right)$ cuando $\lambda$ tienda a $\lambda_{0}$.

OBS.

Se elige generalmente $u_{0}\left(\lambda_{0}\right) \equiv 0$.

HIPOTESIS.

Se supondrá

1) Un problema de bifurcación tiene una solución $u_{0}$ para todos los valores del parámetro $\lambda$ (la cual, sin pérdida de generalidad puede su ponerse idénticamente nula).

2) El problema de bifurcación tiene una linearización de la forma $\mathrm{L}_{1} u=\lambda \mathrm{L}_{2} u$ en que $\mathrm{L}_{1}$ y $\mathrm{L}_{2}$ son operadores lineales acotados en el espacio de Hilbert $\mathrm{H}$.

3) El problema de bifurcación puede escribirse en la forma

$$
\mathrm{I}_{1} u+\mathrm{N}_{1}(u)=\lambda\left(\mathrm{L}_{2}(u)+\mathrm{N}_{2}(u)\right)
$$

donde $\mathrm{N}_{1}, \mathrm{~N}_{2}$ son operadores no lineales estrictamente, en el senti do que 


$$
\begin{aligned}
& \mathrm{N}_{i}(0)=0 \mathrm{y} \\
& \left\|\mathrm{N}_{i}(u)\right\| \leqslant \mathrm{k}\|u\|^{\sigma_{i}}+0\left(\|u\|^{\sigma_{i}}\right), \sigma_{i}>1, i=1,2 \text { para } \\
& \|u\| \text { suficientemente chico. }
\end{aligned}
$$

TEOREMA. (Condición necesaria para los puntos de bifurcación).

Los puntos de bifurcación de la ecuación no lineal

$$
\mathrm{L}_{1} u+\mathrm{N}_{1}(u)+\lambda\left(\mathrm{L}_{2} u+\mathrm{N}_{2}(u)\right)
$$

pueden ocurrir sólo en puntos del espectro de la ecuación linearizada

$$
\mathrm{L}_{1} u=\lambda \mathrm{L}_{2} u
$$

\section{OBSERVACION .}

Este resultado no responde a la cuestión sobre existencia de puntos de bifurcación.

El siguiente ejemplo es una ecuación que no posee puntos de bifurcación y la linearización tiene una cantidad numerable de autovalores:

$$
\begin{aligned}
& \ddot{u}+\lambda\left[u+v\left(u^{2}+v^{2}\right)\right]=0 \\
& \ddot{u}+\lambda\left[v-u\left(u^{2}+v^{2}\right)\right]=0 \\
& u(0)=u(a)=v(0)=v(a)
\end{aligned}
$$




$$
\begin{aligned}
& \ddot{u}+\lambda u=0 \\
& \ddot{v}+\lambda v=0
\end{aligned}
$$

En efecto, si $(u, v)$ es solución, necesariamente

$$
\int_{0}^{a}\left(u^{2}+v^{2}\right)^{2}=0 \text {, de donde } u \equiv 0 \text { y } v \equiv 0
$$

\section{DEMOSTRACION.}

Se supone $\lambda_{0}$ fuera del espectro de la ecuación $L_{1} u=\lambda L_{2} u$, esto es : $L_{1}-\lambda_{0} L_{2}$ es invertible.

Se prueba que si $\left|\lambda-\lambda_{0}\right|$ y $\|u\|$ son suficientemente pequeños entonces la ecuación no lineal tiene solamente la solución trivial $\equiv 0$.

En efecto, sean $T(u)=L_{1} u+N_{1}\left(\omega-\left(L_{2} u+\lambda N_{2}(u)\right)\right.$, $\mathrm{v} u=\mathrm{L}_{1} u-\lambda_{0} \mathrm{~L}_{2} u$. Reordenando en la ecuación siguiente: $T(u)=T(u)-\lambda_{0} L_{2} u+\lambda_{0} L_{2} u$ se obtiene: $T(u)=v u+N_{1}(u)-\lambda N_{2}(u)-\left(\lambda-\lambda_{0}\right) L_{2} u$. Por lo tanto, $\left\|v_{u}\right\| \leqslant\|T(u)\|+\left\|N_{1}(u)\right\|+|\lambda|\left\|N_{2}(u)\right\|+\left|\lambda-\lambda_{0}\right|\left\|I_{2} u\right\|$ Por $\operatorname{ser} \mathrm{V}^{-1} \mathrm{y} \mathrm{L}_{2}$ operadores continuos, existen $\mathrm{k}_{0}>0, \mathrm{k}_{3}>0$ tales que

$$
\mathbf{k}_{0}\|u\| \leqslant\left\|v_{u}\right\|,\left\|\mathrm{L}_{2} u\right\| \leqslant \mathrm{k}_{3}\|u\|
$$

Por hipótesis sobre $\mathrm{N}_{1}, \mathrm{~N}_{2}$ existen $\mathrm{k}_{1}>0, \mathrm{k}_{2}>0$ tales que 
$\left\|\mathrm{N}_{1}(u)\right\| \leqslant \mathrm{k}_{1}\|u\|^{\sigma_{1}}, \quad\left\|\mathrm{~N}_{2}(u)\right\| \leqslant \mathrm{k}_{2}\|u\|^{\sigma_{2}}$

Luego,

$\mathrm{k}_{\mathrm{o}}\|u\| \leqslant\|\mathrm{T}(u)\|+\mathrm{k}_{1}\|u\|^{\sigma_{1}}+\mathrm{k}_{2}|\lambda|\left\|_{u}\right\|^{\sigma_{2}}+\left|\lambda-\lambda_{0}\right| \mathrm{k}_{3}\left\|_{u}\right\|$

Sea $\delta=\min \left\{\left(\frac{k_{0} / 4}{k_{1}}\right)^{\frac{1}{\sigma_{1}-1}},\left(\frac{k_{0} / 4}{\left(1+\left|\lambda_{0}\right|\right) k_{2}}\right)^{\frac{1}{\sigma_{1}-1}}\right\}$

$\mathrm{s}:\|u\| \leqslant \delta$ entonces $\mathrm{k}_{1}\|u\|^{\sigma_{1}} \leqslant \frac{\mathrm{k}_{0}}{4}\|u\| \mathrm{y}$

$k_{2}\left(1+\left|\lambda_{0}\right|\right)\|u\|^{\sigma_{1}} \leqslant \frac{k_{0}}{4}\|u\|$

Luego si $\left|\lambda-\lambda_{0}\right|<\min \left\{1, \frac{\mathrm{k}_{0} / 4}{\mathrm{k}_{3}}\right\}$ y $\|u\| \leqslant \delta$

entonces

$k_{1}\|u\|^{\sigma_{1}}+k_{2}|\lambda|\|u\|^{\sigma_{1}}+\left|\lambda-\lambda_{0}\right| k_{3}\|u\| \leqslant\left(\frac{k_{0}}{4}+\frac{k_{0}}{4}+\frac{k_{0}}{4}\right)\|u\|$

de donde se establece finalmente,

$\|\mathrm{T}(u)\| \geqslant \frac{\mathrm{k}_{0}}{4}\|u\|$

lo cual demuestra que sólo la solución trivial $u \equiv 0$ es posible. 
UN METODO DE PERTURBACION.

REFERENCIA.

M. Krasnosel'skii "Topological methods in the theory of nonlinear integral equations", Pergamon, N.Y. (1964).

Se estudiarán soluciones de norma pequeña de la ecuación no lineal

$$
\mathrm{L}_{1} u+\mathrm{N}_{1}(u)=\lambda\left(\mathrm{I}_{2} u+\mathrm{N}_{2}(u)\right) \ldots \ldots \ldots
$$

en la vecindad de un autovalor real aislado $\lambda_{n}$ de multiplicidad finita del problema linearizado

$$
\begin{aligned}
& \mathrm{I}_{1} u=\lambda \mathrm{L}_{2} u \\
& \text { sea } \lambda=\frac{1}{\mu} \quad \text { y } \quad \lambda_{\mathrm{n}}=\frac{1}{\mu_{\mathrm{n}}} \text { un autovalor real de multiplicidad } \rho
\end{aligned}
$$

de la ecuación linearizada

$$
L_{1} u=\lambda L_{2} u,(I \leqslant \rho<+\infty)
$$

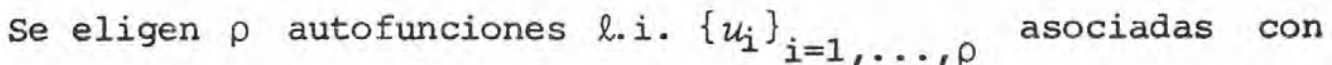
$\lambda_{n}$ tales que

$$
\left(u_{i}, L_{1} u_{j}\right)=\delta_{i j}
$$

\section{HIPOTESIS.}

$L_{1}$ es un operador autoadjunto definido positivo en el espacio de Hilbert $\mathrm{H}$. 
Se denotará $\left[u_{1}, \ldots, u_{\rho}\right]$ el subespacio generado por $u_{1}, \ldots, u_{p}$ y $\mathrm{P}: \mathrm{H} \longrightarrow\left[u_{1}, \ldots, u_{\rho}\right]^{1}$ la proyección de $\mathrm{H}$ sobre el ortogonal de $\left[u_{1}, \ldots u_{\rho}\right]$.

Sea $T(u)=\mu\left(L_{1} u+N_{1}(u)\right)-\left(L_{2} u+N_{2}(u)\right)$

Entonces,

$u$ es solución de la ecuación no lineal $(*) \Longleftrightarrow T(u)=0$

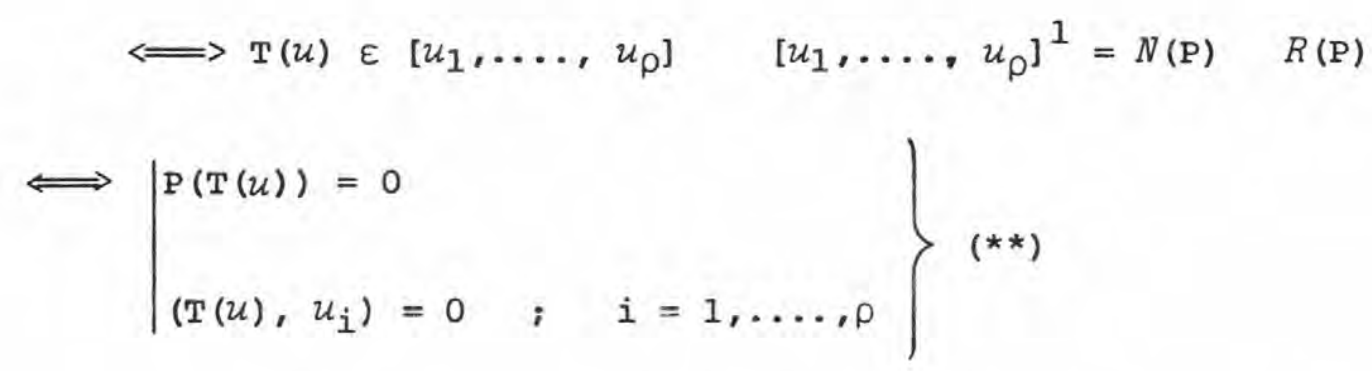

La solución $u$ se puede escribir en forma única:

$u=\mathrm{y}+\sum_{\mathrm{i}=1}^{\rho} \varepsilon_{\mathrm{i}} \quad u_{\mathrm{i}}$ e $\mathrm{H}=R(\mathrm{P})+N(\mathrm{P})$

donde $y \in\left[u I, \ldots, u_{\rho}\right]^{1}, G_{i} \in \mathbb{R}, i=1, \ldots, \rho$

\section{IEATA .}

Bajo las hipótesis hechas, si a) $\mathrm{N}_{1}(u)=0$ o bien b) $\mathrm{N}_{2}(u)=0$, la primera ecuación de (***) tiene una única solución "y" en función de $E_{1}, \ldots, E_{\rho}$

Además, se tiene la siguiente estimación para la solución $\mathrm{y}=\mathrm{y}\left(E_{1}, \ldots, E_{\rho}\right):$ 


$$
\|\mathrm{y}\| \leqslant \mathrm{k}_{3}|E|^{\rho_{i}} \text { donde }|E|=\left|E_{1}\right|+\ldots .+\left|E_{\rho}\right|
$$

$\mathrm{y} \mathrm{k}_{3}>0$ es independiente de $\left(E_{1}, \ldots, E_{\rho}\right)$

DEMOSTRACION DEL LEMA.

La primera ecuación de $(* * *)$ es:

$$
y=\left(\mu L_{1}-L_{2}\right)^{-1} \quad P\left(-\mu N_{1}(u)+N_{2}(u)\right)
$$

Por analogía basta considerar uno de los dos casos.

Consideremos el caso a) $\mathrm{N}_{1} \equiv 0$ lo que reduce la ecuación a:

$$
\mathrm{y}=\left(\mu \mathrm{L}_{1}-\mathrm{L}_{2}\right)^{-1} \mathrm{P}\left(\mathrm{N}_{2}\right)
$$

y la ecuación no lineal a

$$
L_{1} u=\lambda\left[L_{2}(u)+N_{2}(u)\right]
$$

Basta demostrar que la aplicación definida por

$$
\mathrm{T}_{E}(\mathrm{z})=\left(\mu \mathrm{L}_{1}-\mathrm{L}_{2}\right)^{-1} \mathrm{P}\left(\mathrm{N}_{2}\left(\mathrm{z}+\sum_{i=1}^{\rho} E_{i} u_{i}\right)\right)
$$

es una contracción.

$$
\text { Sean } y, \bar{y} \varepsilon H, u=y+\sum E_{i} u_{i}, \bar{u}=\bar{y}+\sum E_{i} u_{i} \text {, }
$$

entonces

$$
\mathrm{T}_{E}(\mathrm{y})-\mathrm{T}_{E}(\overline{\mathrm{y}})=\left(\mu \mathrm{L}_{1}-\mathrm{L}_{2}\right)^{-1} \mathrm{P}\left(\mathrm{N}_{2}(u)-\mathrm{N}_{2}(\bar{u})\right)
$$


Por hipótesis

$$
\begin{aligned}
& \mathrm{N}_{2}(u)-\mathrm{N}_{2}(\bar{u})=\mathrm{P}_{2}(u)-\mathrm{P}_{2}(\bar{u})+\mathrm{R}_{2}(u)-\mathrm{R}_{2}(\bar{u}) \\
& \text { Por lo tanto } \\
& \left\|\mathrm{T}_{E}(\mathrm{y})-\mathrm{T}(\overline{\mathrm{y}})\right\| \leqslant\left\|\left(\mu \mathrm{L}_{1}-\mathrm{L}_{2}\right)^{-1}\right\|\left\|\mathrm{N}_{2}(u)-\mathrm{N}_{2}(\bar{u})\right\| \\
& \left\|\mathrm{N}_{2}(u)-\mathrm{N}_{2}(\bar{u})\right\| \leqslant \mathrm{k}_{0}\left\{\|u\|^{\rho_{2-1}}+\|u\|^{\rho_{2-1}}\right\}\|u-\bar{u}\|+\mathrm{k}_{1} \\
& \left\{\|u\|^{\rho_{2}}+\|\bar{u}\|^{\rho_{2}}\right\}\|u-\bar{u}\|
\end{aligned}
$$

Como

$$
\|u\|=\| y+\left[E_{i} u_{i}\|\leq\| y\left\|+|E| \sum\right\| u_{i} \|\right.
$$

escogiendo $\|y\| \leqslant|E|,\|\bar{y}\| \leqslant|E|,|E|$ suficientemente pequeño se tiene:

$$
\|u\|^{\rho_{2}} \leqslant\|u\|^{\rho_{2-1}} \leqslant k_{2}|E|^{\rho_{2-1}},\|\bar{u}\|^{\rho_{2}} \leqslant \overline{\mathrm{k}}_{2}|E|^{\rho_{2-1}}
$$

de donde concluir,

$$
\begin{aligned}
& \left\|\mathrm{T}_{E}(\mathrm{y})-\mathrm{T}_{E}(\overline{\mathrm{y}})\right\| \leqslant \mathrm{k}|E|^{\rho_{2-1}}\|\mathrm{y}-\overline{\mathrm{y}}\| \\
& \quad \text { Luego, si }|E| \text { es suficientemente pequeño existe } \alpha<1 \text { tal que } \\
& \left\|\mathrm{T}_{E}(\mathrm{y})-\mathrm{T}_{E}(\overline{\mathrm{y}})\right\| \leqslant \alpha\|\mathrm{y}-\overline{\mathrm{y}}\|
\end{aligned}
$$

de lo cual se concluye que existe un único $y=T_{E}(y)$ punto fijo solución de la primera ecuación de $(* * *)$. 
69

Además, como $\|y\| \leqslant|E|$ se tiene:

$$
\begin{aligned}
\|y\| & =\left\|\mathrm{T}_{E}(\mathrm{y})\right\| \\
& =\left\|\mathrm{T}_{E}(\mathrm{y})-\mathrm{T}_{\mathrm{O}}(\mathrm{y})+\mathrm{T}_{\mathrm{O}}(\mathrm{y})\right\| \\
& \leqslant\left\|\mathrm{T}_{E}(\mathrm{y})-\mathrm{T}_{\mathrm{O}}(\mathrm{y})\right\|+\left\|\mathrm{T}_{\mathrm{O}}(\mathrm{y})\right\| \\
& \leqslant\left\|\left(\mu \mathrm{L}_{1}-\mathrm{L}_{2}\right)^{-1}\right\|\left\|\mathrm{N}_{2}(u)-\mathrm{N}_{2}(\mathrm{y})\right\|+\left\|\left(\mu \mathrm{L}_{1}-\mathrm{L}_{2}\right)^{-1}\right\|\left\|\mathrm{N}_{2}(\mathrm{y})\right\|
\end{aligned}
$$

Se recuerda

$$
\begin{aligned}
& \left\|\mathrm{N}_{2}(\mathrm{y})\right\| \leqslant \mathrm{k}_{0}\|\mathrm{y}\|^{\rho_{2}}+\mathrm{k}_{1}\|\mathrm{y}\|^{\rho_{2+1}} \leqslant \mathrm{k}_{3}|E|^{\rho_{2}} \text { si }|E|<1 \\
& \left\|N_{2}(u)-N_{2}(y)\right\| \leqslant k_{0}\left\{\|u\|^{\rho_{2-1}}+\|y\|^{\rho^{2-1}}\right\}\|u-y\|+k_{1} \\
& \left\{\|u\|^{\rho_{2}}+\|y\|^{\rho_{2}}\right\}\|u-y\| \\
& u-\mathrm{y}=\sum E_{\mathrm{i}} u_{\mathrm{i}} \Longrightarrow\|u-\mathrm{y}\| \leqslant \mathrm{k}_{4}|E| \\
& \|y\|^{\rho_{2-1}} \leqslant|E|^{\rho_{2-1}},\|y\|^{\rho_{2}} \leqslant|E|^{\rho_{2}} \\
& \|u\| \leqslant\|y\|+|E| \sum\left\|u_{i}\right\| \leqslant\left(1+\mathrm{k}_{5}\right)|E| \\
& \text { Por lo tanto } \\
& \|u\|^{\rho_{2}} \leqslant \mathrm{k}_{6}|E|^{\rho_{2}},\|u\|^{\rho_{2-1}} \leqslant \mathrm{k}_{7}|E|^{\rho_{2}-1} \\
& \text { Luego existe } \mathrm{k} \text { independiente de } E_{i}, i=1, \ldots, \rho \text { tal que } \\
& \|y\| \leqslant k \quad|E|^{\rho_{2}}
\end{aligned}
$$


BIFURCACION DE UN AUTOVALOR SIMPLE $(\rho=1)$

Se hace un análisis completo de la existencia, multiplicidad y teoría espectral para la ecuación operacional

$$
\mathrm{L}_{1} u+\mathrm{N}_{2}(u)=\lambda\left(\mathrm{I}_{2} u+\mathrm{N}_{2}(u)\right)
$$

bajo las hipótesis hechas.

TEOREMA DE EXISTENCIA Y MULTIPLICIDAD.

Además de las hipótesis hechas se supondrá que $\left(\mathrm{P}_{i} u_{1}, u_{1}\right) \neq 0$ $(j=1,2)$ en que $u_{1}$ es la autofunción normalizada de la ecuación $\mathrm{I}_{1} u=\lambda_{\mathrm{n}} \mathrm{L}_{2} u$. Entonces en el caso a) o en el caso b), la ecuación tiene una única familia a un parámetro de soluciones de norma pequeña con autovalor $\lambda$ cerca de $\lambda_{n}$.

\section{TEOREMA ESPECTRAL,}

Bajo las hipótesis del teorema de existencia, el comportamiento local de las familias a un parámetro de soluciones es cualitativamen te descrito por la paridad de los números $\rho_{1}, \rho_{2}$, el signo de las expre siones $\left(P_{i} u_{1}, u_{1}\right)(i=1,2)$ y el signo de $\lambda_{n}$.

Sustituyendo en el sistema $(* *)$ de $(\rho+1)$ - ecuaciones, se obtiene el sistema de incógnitas y, i :

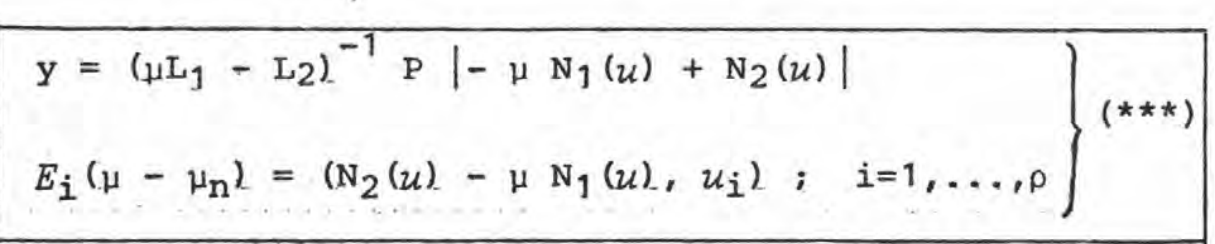




\section{ORTENTACION .}

En lo que sigue interesarán sólo soluciones de norma pequeña, es decir, se supondrá que tanto $\|y\|$ como los números $E_{i}$ son pequeños comparados con 1 .

HIPOTESIS DE SIMPLIFICACION.
a). $\mathrm{N}_{1} \equiv 0$
o bien
b) $\mathrm{N}_{2} \equiv 0$

\section{HIPOTESIS.}

$$
\begin{aligned}
& \text { Para }\|u\|,\|v\| \quad \text { suficientemente pequeño. } \\
& \mathrm{N}_{i}(u)=\mathrm{P}_{i}(u)+\mathrm{R}_{i}(u) \quad i=1,2
\end{aligned}
$$

donde $\mathrm{P}_{i}$ es un operador homogéneo de grado $\rho_{i}>1$, es decir,

$$
\begin{gathered}
\mathrm{P}_{i}(\sigma u)=\sigma^{\rho} \mathrm{P}_{i}(u), \rho_{i}>1 \text { y donde } \\
\left\|\mathrm{P}_{i}(u)-\mathrm{P}_{i}(v)\right\| \leqslant \mathrm{k}_{0}\left\{\|u\|^{\rho}{ }^{\rho-1}+\|v\|^{\rho-1}\right\}\|u-v\| \\
\left\|\mathrm{R}_{i}(u)-\mathrm{R}_{i}(v)\right\| \leqslant \mathrm{k}_{1}\left\{\|u\|^{\rho}+\|v\|^{\rho}\right\}\|u-v\|
\end{gathered}
$$

en que $\mathrm{k}_{0}, \mathrm{k}_{1}$ son constantes independientes de $u \mathrm{y} v$. 
NOTA.

$$
N_{i}(u)=P_{i}(u)+R_{i}(u), \quad i=1,2
$$

$P_{i}$ operador homogéneo de grado $\rho_{i}: P_{i}(\sigma)=\sigma^{\rho_{i}} u$

Como $P_{i}(0)=0, N_{i}(0)=0$, entonces $R_{i}(0)=0$

$$
\begin{aligned}
& \left\|\mathrm{P}_{i}(u)-\mathrm{P}_{i}(v)\right\| \leqslant \mathrm{k}_{0}\left\{\|u\|^{\rho_{i-1}}+\|v\|^{\rho_{i-1}}\right\}\|u-v\| \\
& \left\|\mathrm{R}_{i}(u)-\mathrm{R}_{i}(v)\right\| \leqslant \mathrm{k}_{1}\left\{\|u\|^{\rho}+\|v\|^{\rho}\right\}\|u-v\|
\end{aligned}
$$

en particular

$$
\begin{aligned}
& \left\|\mathrm{P}_{i}(u)\right\| \leqslant \mathrm{k}_{0}\|u\|^{\rho_{i}} \\
& \left\|\mathrm{R}_{i}(u)\right\| \leqslant \mathrm{k}_{1}\|u\|^{\rho_{i+1}}=\mathrm{k}_{1}\|\|^{\rho_{i}}\|u\| \\
& \left\|\mathrm{N}_{i}(u)\right\| \leqslant \mathrm{k}_{0}\|u\|^{\rho_{i}}+\mathrm{k}_{1}\|u\|^{\rho_{i+1}}
\end{aligned}
$$

DEMOSTRACION. (Del Teorema de Existencia)

Se recuerda que $\rho=1$. Se considera el caso a) $\mathrm{N}_{1} \equiv 0$.

Por el lema anterior basta considerar la segunda ecuación del sistema $(* * *)$.

$$
E\left(\mu-\mu_{n}\right)=\left(N_{2}(u), u_{1}\right) \quad \ldots \ldots
$$

para $|E|$ suficientemente pequeño. 
Se observa:

$$
\begin{aligned}
\left(\mathrm{N}_{2}(u), u_{1}\right) & =\left(\mathrm{P}_{2}(u)+\mathrm{R}_{2}(u), u_{1}\right) \\
& =\left(\mathrm{P}_{2}\left(E u_{1}\right), u_{1}\right)+\left(\mathrm{P}_{2}(u)-\mathrm{P}_{2}\left(E u_{1}\right), u_{1}\right)+\left(\mathrm{R}_{2}(u), u_{1}\right)
\end{aligned}
$$

en que, por ser $\mathrm{P}_{2}$ homogéneo:

$$
\left(\mathrm{P}_{2}\left(E u_{1}\right) ; u_{1}\right)=E^{\rho_{2}}\left(\mathrm{P}_{2}\left(u_{1}\right), u_{1}\right)
$$

De la hipótesis sobre $\mathrm{P}_{2}$ y $\mathrm{R}_{2}$ :

$$
\begin{aligned}
& \left|\left(R_{2}(u), u_{1}\right)\right| \leqslant\left\|R_{2}(u)\right\|\left\|u_{1}\right\| \leqslant k\|u\|^{\rho} 2+1 \\
& \left|\left(\mathrm{P}_{2}(u)-\mathrm{P}_{2}\left(E u_{1}\right), u_{1}\right)\right| \leqslant\left\|\mathrm{P}_{2}(u)-\mathrm{P}_{2}\left(E u_{1}\right)\right\|\left\|u_{1}\right\|
\end{aligned}
$$

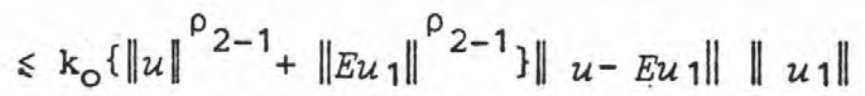

$$
\begin{aligned}
& \text { Pero }\|u\| \leqslant \mathrm{k}_{2}|E|, u=\mathrm{y}+E u_{1},\|\mathrm{y}\| \leqslant \mathrm{k}_{3}|E|^{\rho_{2}} \text { entonces } \\
& \left|\left(\mathrm{P}_{2}(u)-\mathrm{P}_{2}\left(E u_{1}\right), u_{1}\right)\right| \leqslant \mathrm{k}|E|^{2 \rho_{2-1}} \leqslant \mathrm{k}|E|^{\rho_{2+1}}
\end{aligned}
$$

Luego

$$
E\left(\mu-\mu_{n}\right)=\left(\mathrm{P}_{2}\left(E u_{1}\right), u_{1}\right)(1+0(1))
$$

Por lo tanto, para $|E|$ suficientemente pequeño, las soluciones de (1) puede ser completamente descritas estudiando las soluciones de la ecuación

$$
\left(\mu-\mu_{n}\right)=\left(P_{2}\left(E u_{1}\right), u_{1}\right)=E^{\rho_{2}}\left(P_{2}\left(u_{1}\right), u_{1}\right)
$$


que para $E \neq 0$ es equivalente a:

$$
E^{1-\rho_{2}}\left(\mu-\mu_{n}\right)=\left(P_{2}\left(u_{1}\right), u_{1}\right)=\text { constante }
$$

Se recuerda $u=y+E u_{1}$ en que $y=y(E)$

Luego para $|E|$ suficientemente pequeño, las soluciones de $L_{1} u=\lambda\left(L_{2} u+N_{2}(u)\right)$ cerca de $\lambda=\lambda_{n}$ pueden ser descritas como una familia a 1-parámetro $u(E)$ 冈 $u(E) \longrightarrow u_{0} \equiv 0$ cuando $E \longrightarrow 0$.

Note que si $E=0$ necesariamente $y=0$, por lo tanto la ecua ción no lineal correspondiente a $\lambda=\lambda_{n}$ tiene sólo la solución trivial $u_{0} \equiv 0$.

DEMOSTRACION DEL TEOREMA ESPECTRAL.

Para el caso a) bastaba observar la ecuación

$$
E\left(\mu-\mu_{n} L=E^{\rho} 2\left(P_{2}\left(u_{1}\right), u_{1}\right)\right.
$$

y para el caso b)

$$
E\left(\mu-\mu_{n}\right)=-\mu E^{\rho_{1}}\left(\mathrm{P}_{1}\left(u_{1}\right), u_{1}\right)
$$

Por lo tanto la dependencia cualitativa de $\lambda$ sobre $E$ cerca de $\lambda=\lambda_{n}$ depende de la paridad de $\rho_{1} \sigma \rho_{2}$ y de los signos de los factores. 
Caso a). $\quad \lambda_{n}>0$

$\rho_{2}$ impar signo factor $<0 \Longrightarrow$ hay dos soluciones para $\lambda<\lambda_{n}$, no

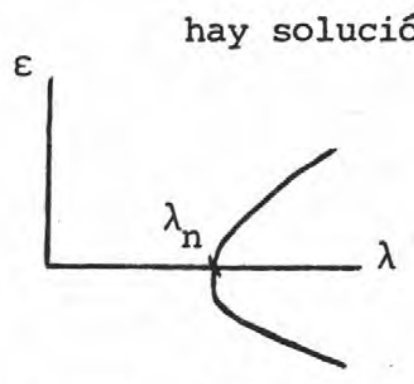

signo factor $>0 \Longrightarrow$ situación al revés.

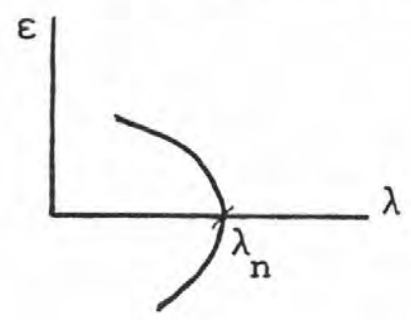

$\rho_{2}$ par signo factor $>0 \Longrightarrow$ hay solución única para todo $\lambda$.
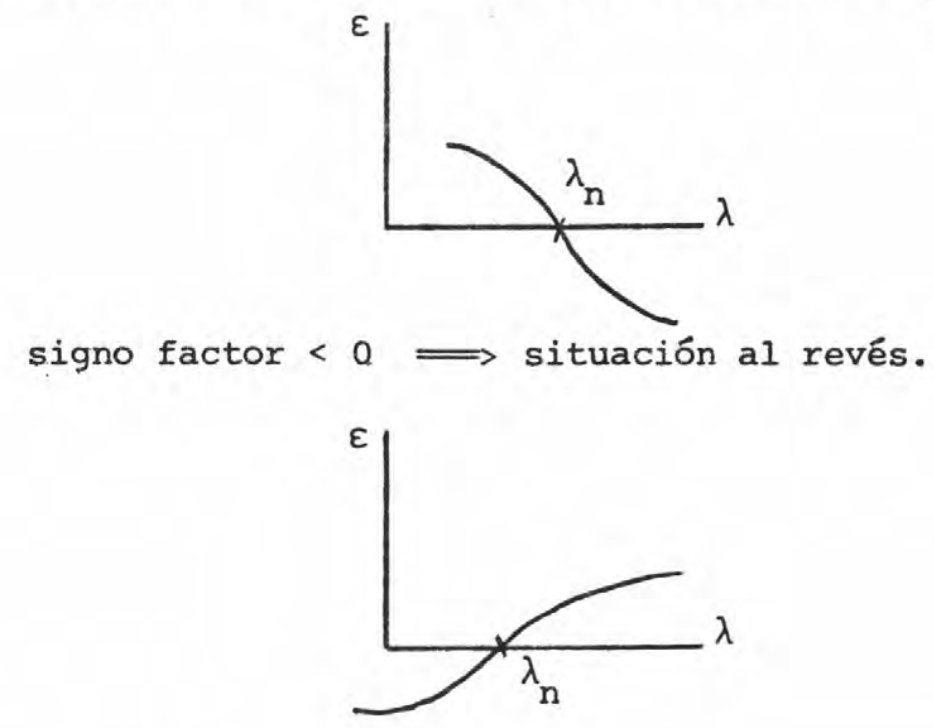

Caso bL. $\quad \lambda_{n}>0$

por el signo negativo, las conclusiones son las contrarias de las del caso a). 
EJEMPLO.

Sea $G$ un dominio acotado en $\mathbb{R}^{3}$ y considera el PVF

$$
\begin{aligned}
& \Delta u+\lambda(\mathrm{e}(\mathrm{x}) u+f(u))=0 \text { en } \mathrm{G} \\
& u=0 \text { en } \partial \mathrm{G}
\end{aligned}
$$

Aqui $f(u)=\alpha_{q} u^{q}+\alpha_{q+1} u^{q+1}+\ldots$. (q entero $>1$

$$
\alpha_{q} \neq 0 \text { ) }
$$

$y e(x)>0$.

Se tiene que $\lambda_{n}>0, \lambda_{1}$ necesariamente de multiplicidad 1 $y\left(P_{2} u, u\right)=\int_{G} \alpha_{q} u^{q+1}$. Si q es impar $y \alpha_{q}>0 \sigma \alpha_{q}<0$ se apli can los resultados del estudio. 